\title{
Light yield of Kuraray SCSF-78MJ scintillating fibers for the GlueX Barrel Calorimeter
}

\author{
T.D. Beattie ${ }^{\mathrm{a}, *}$, A.P. Fischer, ${ }^{\mathrm{a}, 1}$, S.T. Krueger ${ }^{\mathrm{a}}$, G.J. Lolos ${ }^{\mathrm{a}}$, Z. Papandreou ${ }^{\mathrm{a}, *}$, \\ E.L. Plummer ${ }^{a}, 1$, A.Yu. Semenov ${ }^{\text {a }}$, I.A. Semenova ${ }^{a}$, L.M. Sichello ${ }^{a, 1}$, \\ L.A. Teigrob ${ }^{a}$, E.S. Smith ${ }^{\mathrm{b}}$ \\ ${ }^{a}$ Department of Physics, University of Regina, Regina, SK, S4S 0A2, Canada \\ ${ }^{b}$ Thomas Jefferson National Accelerator Facility (Jefferson Laboratory), Newport News, \\ Virginia, 23606, USA
}

\begin{abstract}
Over three quarters of a million 1-mm-diameter 4-m-long Kuraray double-clad SCSF-78MJ (blue-green) scintillating fibers have been used in the construction of the GlueX electromagnetic barrel calorimeter for the Hall D experimental program at Jefferson Lab. The quality of a random sample of 4,750 of these fibers was evaluated by exciting the fibers at their mid point using a ${ }^{90} \mathrm{Sr}$ source in order to determine the light yield using a calibrated vacuum photomultiplier as the photosensor. A novel methodology was developed to extract the number of photoelectrons detected for measurements where individual photoelectron peaks are not discernible. The average number of photoelectrons from this sample of fibers was $9.17 \pm 0.6$ at a source distance of $200 \mathrm{~cm}$ from the PMT.
\end{abstract}

Key words: scintillating fibers, light yield, number of photoelectrons PACS: 29.40.Mc, 29.40.Vj

\section{Project background}

The results reported in this paper are a subset of a larger study undertaken to evaluate the performance and quality control of Kuraray double-clad SCSF$78 \mathrm{MJ}$ scintillating fibers ${ }^{2}$ to be used in the electromagnetic barrel calorimeter (BCAL) for the GlueX project. Measurements on the spectral response and attenuation length of the fibers have been published previously [1. Herein are the results on the light yield measured following stimulation of the fibers at their midpoint using a ${ }^{90} \mathrm{Sr}$ source.

\footnotetext{
This work was carried out as part of the GlueX Project at Jefferson Lab.

*Corresponding author. Tel.: +1 306585 5379; fax: +1 3065855659

Email addresses: zisis@uregina.ca (Z. Papandreou)

${ }^{1}$ Student has graduated.

${ }^{2}$ Kuraray America Inc., Houston, TX, USA (www.kuraray-am.com)
} 
GlueX aims to elucidate the phenomenon of confinement in Quantum Chromodynamics by searching for exotic hybrid mesons that arise from photoproduction at $\sim 9 \mathrm{GeV}[2$, an effort that requires a hermetic detector. A major subsystem deployed inside this detector's superconducting solenoid is the BCAL, which will primarily detect photons resulting from $\pi^{0} \rightarrow \gamma \gamma$ and $\eta \rightarrow \gamma \gamma$ decays between $60 \mathrm{MeV}$ and $2.5 \mathrm{GeV}, 11^{\circ}-126^{\circ}$ in polar angle, and $0^{\circ}-360^{\circ}$ in azimuthal angle.

The BCAL consists of 48 modules, each with a trapezoidal cross section, forming a $390 \mathrm{~cm}$ long (hollow) cylinder having inner and outer radii of $65 \mathrm{~cm}$ and $90 \mathrm{~cm}$, respectively. This detector is an electromagnetic sampling calorimeter comprised of a lead and scintillating fiber matrix, with the fibers running parallel to the cylindrical axis of the detector. Each module consists of 185 layers of corrugated lead sheets, each of $0.5 \mathrm{~mm}$ thickness, and 184 layers of 1-mm-diameter double-clad fibers bonded in $0.5 \mathrm{~mm}$-deep grooves of the lead sheets using BC-600 optical epoxy ${ }^{3}$ This geometry results in $\sim 14,225$ fibers per module.

\section{Fiber overview}

Kuraray SCSF-78MJ fibers $4^{4}$ were selected for the BCAL, as they have an attenuation length near $4 \mathrm{~m}$. It is also important that the fibers yield the highest possible amount of light, as this feature impacts the energy and timing resolutions as well as the detection threshold. The light transmitted by the fibers will be collected by acrylic light guides before being read out by largearea Hamamatsu S12045(X) multi pixel photon counter 5 (MPPCs), also known as silicon photomultipliers (SiPMs). The performance of these devices has been published by our collaboration [3, 4, 5].

The fibers were delivered in 29 shipments, which included 707 fiber "preform batches" (or "lots"), with each lot averaging about 1,100 fibers. Each crate was packed with cardboard boxes, with each box containing two pouches with fibers from two lots, while each pouch was subdivided into nine to twelve packs. About $0.6 \%$ of the fibers were tested from each shipment, with one fiber being randomly selected from each pack.

Data were collected from fibers with their far end polished and blackened using black matte enamel paint (used for scale model painting) for shipments 13. Following those measurements, it was decided to leave the far ends as factory cut and only blacken them for shipments 4-29 in order to simplify the preparation process and to be able to compare our results directly to Kuraray's measurements. As a result, shipments 1-3 (Lots 1-95) were excluded from all comparisons below.

\footnotetext{
${ }^{3}$ St. Gobain Crystals \& Detectors, Hiram, OH 44234, USA (www.bicron.com)

${ }^{4}$ Kuraray Plastic Scintillating Fibers (kuraraypsf.jp/psf/sf.html)

${ }^{5}$ Hamamatsu Corporation, Bridgewater, NJ 08807, USA (www.hamamatsu.com)
} 
The specific parameter tested and reported herein is the number of photoelectrons, $N_{p e}$, measured using a calibrated vacuum photomultiplier (PMT) following the stimulation of the fiber by a ${ }^{90} \mathrm{Sr}$ source at its midpoint, $200 \mathrm{~cm}$ from its end. Contract specifications with the manufacturer included the requirement of $N_{p e}>4.5$ under these conditions and $R M S \leq 15 \%$ for the entire fiber sample. The measurements of nearly 4,750 fibers afforded the determination of the light yield (in terms of $N_{p e}$ ) with statistical confidence.

\section{Experimental and analysis methodology}

\subsection{Experimental setup}

The experimental methodology was established using a fiber from a firstarticl $e^{6}$ group of fibers: The setup is sketched in Figure 1 Both ends of the fiber were polished and one was blackened. The fiber was then inserted in a dedicated measuring station contained in a 4.5-m-long dark box. A Hamamatsu R329-02 calibrated photomultiplier with a standard progressive voltage divider was used to measure the light from the fiber. A small dab of optical greas 7 was applied to the non-blackened polished end of the fiber before coupling it to the PMT using a piece of machined acrylic as a fiber guide. Even though this was done carefully, measurements carried out at the start of the project showed that differences in light transmission of up to $2-3 \%$ percent can arise from the combined polishing and subsequent coupling (using optical grease) of fiber ends.

The fiber was stimulated using a ${ }^{90} \mathrm{Sr}$ radioactive source, which was placed within a custom-designed lead collimator and positioned over the center point of the fiber, approximately $200 \mathrm{~cm}$ from the PMT's end. The collimator consisted of a $4 \times 4 \mathrm{~cm}^{2} \mathrm{~Pb}$ block, having an $8 \mathrm{~mm}$ diameter partial bore to house the source. Located below the source was a slit having dimensions $13(h) \times 0.5(w) \times$ $8(l) \mathrm{mm}^{3}$ and a $1 \times 1 \mathrm{~mm}^{2}$ groove along the bottom of the collimator for accurate placement and illumination of the tested fiber, the groove being parallel to the 8-mm-slit side. The collimator included a lid, since it was determined that a small amount of "backsplash" activity resulted from source electrons striking the lid of the dark box. A $\sim 9 \mathrm{~cm}$-long scintillator counter was coupled to a Burle 8575 PMT (at $1700 \mathrm{~V}$ ) and was placed directly below the fiber, thus sandwiching the fiber between it and the source/collimator above. This counter provided the trigger for the data acquisition at a rate of approximately $1 \mathrm{kHz}$.

Care was exercised in the setting of the discriminator threshold. Measurements were also taken with the ${ }^{90} \mathrm{Sr}$ removed and with the source and trigger counter moved away from the fiber in order to determine the PMT pedestal and the random triggers, the latter being indeed random and measured with an accuracy better than $1 \%$. The stability of the pedestal position was monitored on a run-by-run basis.

\footnotetext{
${ }^{6}$ Three groups of 55 fibers each were produced and distributed between Kuraray, Jefferson Lab and the University of Regina for quality evaluation prior to production.

${ }^{7}$ EJ-550 Silicone Optical Grease, ELJEN Technology, (www.eljentechnology.com/)
} 


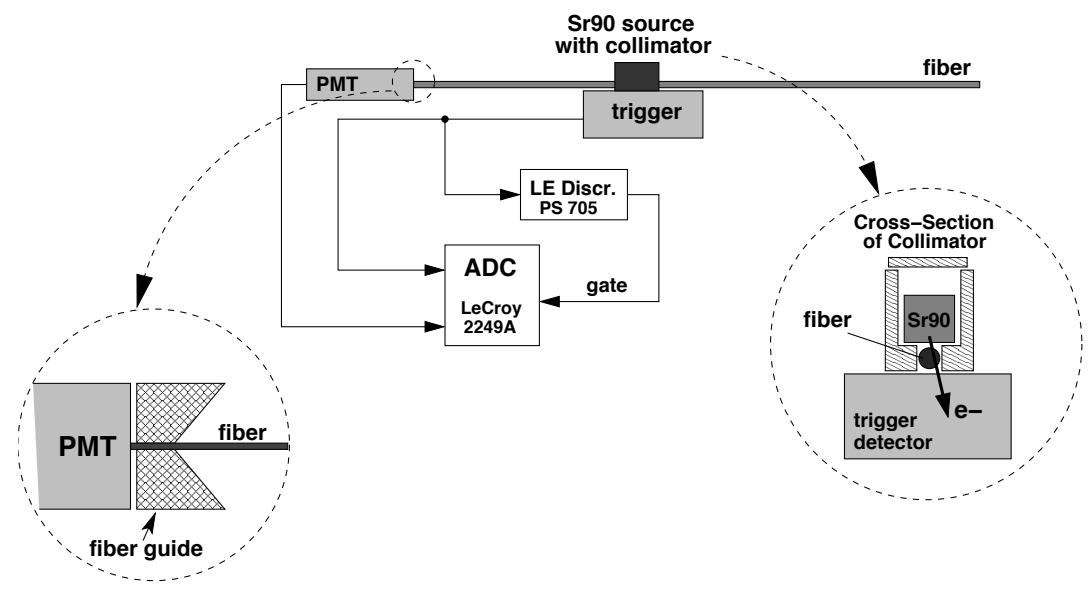

Figure 1: The geometrical setup used to measure the number of photoelectrons from the fibers is sketched (not to scale); the PMTs, fiber and source are enclosed in a $4.5 \mathrm{~m}$ long dark box. The inset on the left shows the acrylic piece that is used to guide the fiber in contacting the PMT, and the inset on the right shows the cross sectional view of the lead collimator and its lid containing the ${ }^{90} \mathrm{Sr}$ radioactive source. The circuit diagram is also presented. The reader is referred to the text for more details.

\subsection{Analysis method}

ADC spectra for each fiber were accumulated from both the trigger and fiber PMT and were stored using our CAMAC-based data acquisition system. To first order, the analysis method relies on the Poisson statistics of the ADC distribution from the fiber PMT, whereby $N_{p e}$ is extracted from the ratio of the square of the ADC distribution's mean to the square of its RMS. Care is required in the determination of the latter, and the understanding of the PMT's gain is a key step in the process.

The width of the measured ADC spectra originates from fluctuations in the number of photons, photoelectons and variations in the energy deposited in the fiber. To properly address these effects, we simulated the energy deposition from the ${ }^{90} \mathrm{Sr}$ electrons in a realistic model of the measurement setup using the FLUKA 2008.3 program 7 . It should be emphasized that the exact dimensions of the collimator and fiber positioning were used in the simulation. The simulated, Birks-corrected energy depositions in the fiber and trigger scintillator were recorded on an event-by-event basis, and the estimated threshold on the trigger counter was applied in order to obtain the simulated spectrum, $F(E)$, shown in Figure 2. 


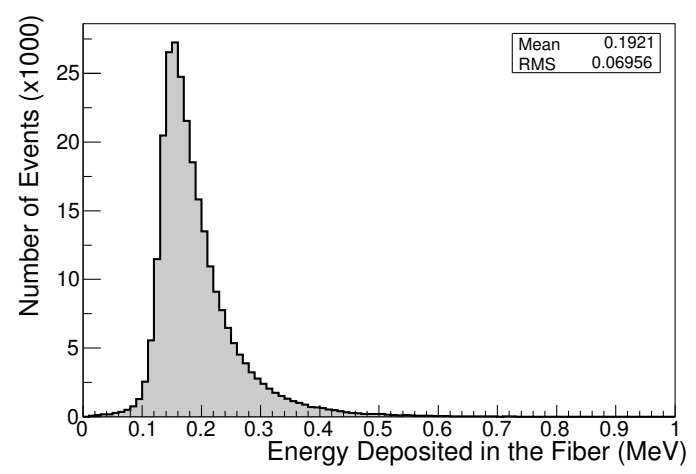

Figure 2: The simulated distribution of the energy deposition in a fiber is shown. The simulated mean energy deposition was $\sim 0.19 \mathrm{MeV} /$ fiber, corresponding to traversal of $\beta$ particles from the ${ }^{90} \mathrm{Sr} /{ }^{90} \mathrm{Y}$ mother/daughter pair past the collimator and through the 1-mm fiber. The simulated geometry mirrors the experimental setup as described in the text.

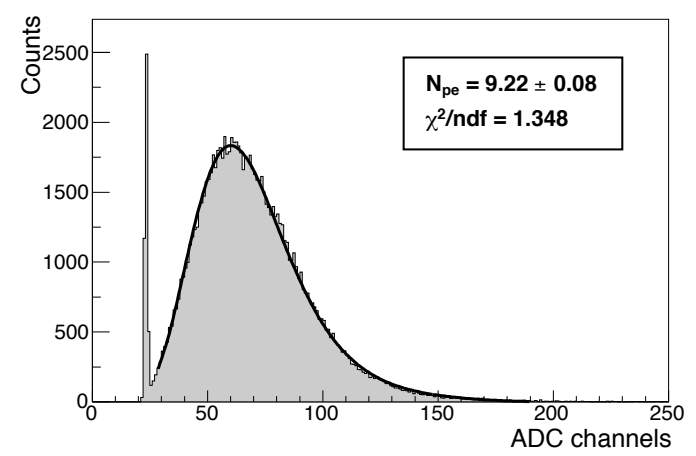

Figure 3: A typical photoelectron spectrum is shown when both the ${ }^{90} \mathrm{Sr}$ source and the trigger counter were positioned $200 \mathrm{~cm}$ from the fiber's end. The spectrum is fit with a Poisson function with the extracted mean number of photoelectrons and the $\chi^{2}$ per d.o.f. displayed in the inlay. Details on the fitting function are described in the text.

A sample ADC spectrum from the fiber PMT is shown in Figure 3 Using the simulated $F(E)$ functions, experimental, pedestal-subtracted ADC spectra from the PMT were fit (solid line in Figure 3 ) to the function :

$$
f(x)=k_{0} \int_{0}^{E_{\max }} d E\left(F(E) \sum_{N_{\gamma}=0}^{N_{\gamma}^{\max }} \mathcal{P}\left(N_{\gamma}, k_{1} E\right) \cdot \beta\left(N_{\gamma}\right)\right)
$$

where $\beta\left(N_{\gamma}\right)$ is determined from

$$
\beta\left(N_{\gamma}\right)=\sum_{N_{p e}=0}^{N_{\gamma}} \mathcal{B}\left(N_{p e}, N_{\gamma}, \epsilon_{q}\right) \mathcal{G}\left(x, k_{2} N_{p e}, k_{2} \sigma_{p e}\right)
$$


and $k_{0}, k_{1}$ and $k_{2}$ are free parameters in the fit, with $k_{0}$ accounting for the overall scale of the spectrum. The Poisson distribution $\mathcal{P}\left(N_{\gamma}, k_{1} E\right)$ - with the expected value $k_{1} E$ - is used in equation 1 to describe the distribution of the photons on the PMT's photocathode that corresponds to energy $E$ deposited in the fiber; the factor $k_{1}$ combines the energy-to-photon conversion factor as well as the capture and attenuation of photons in the fiber.

The conversion of photons to photoelectrons is described in equation 2 as the Binomial distribution $\mathcal{B}\left(N_{p e}, N_{\gamma}, \epsilon_{q}\right)$, where the probability of the conversion is captured as the convoluted quantum efficiency (CQE) of the PMT's photocathode, $\epsilon_{q}$. This was determined by convoluting the manufacturer's QE data with the Kuraray's SCSF-78MJ fiber's spectrum as a function of wavelength; details are presented in reference [11. The resulting CQE was determined to be $0.198 \pm 0.010$ — with an assigned $5 \%$ uncertainty on this value - in order to account for the possible difference between the integrated quantum efficiency (measured by Hamamatsu) and the local CQE at the center of photocathode where the fiber was coupled and where the CQE is the highest. It should be noted that this uncertainty propagates to $\approx 1 \%$ uncertainty in the final result, and is one of the dominant sources of systematic error in this method. For practical reasons (manageable analysis processing time), the Binomial distribution, $\mathcal{B}\left(N_{p e}, N_{\gamma}, \epsilon_{q}\right)$, was approximated by a corresponding Poisson distribution, $\mathcal{P}\left(N_{p e}, \epsilon_{q} N_{\gamma}\right)$, having the expected value $\epsilon_{q} N_{\gamma}$, such that $\beta\left(N_{\gamma}\right)$ becomes:

$$
\beta\left(N_{\gamma}\right) \approx \sum_{N_{p e}=0}^{N_{\gamma}} \mathcal{P}\left(N_{p e}, \epsilon_{q} N_{\gamma}\right) \mathcal{G}\left(x, k_{2} N_{p e}, k_{2} \sigma_{p e}\right)
$$

The process of electron multiplication through the PMT's dynode chain is captured in the Gaussian distribution $\mathcal{G}\left(x, k_{2} N_{p e}, k_{2} \sigma_{p e}\right)$ in equation 2. There, $k_{2}$ includes the conversion from $N_{p e}$ to ADC channels and is proportional to the PMT gain. The ratio of the mean value of the ADC spectrum (following pedestal subtraction) to $k_{2}$ provides the average $N_{p e}$ from the fiber (viz., the final result). The width of the PMT gain function, $\sigma_{p e}$, corresponding to $N_{p e}$, is estimated using the formula (see [8, 9]):

$$
\sigma_{p e}^{2} \approx N_{p e} \frac{g^{m}-1}{g^{m}(g-1)} \approx N_{p e} \frac{1}{g-1},
$$

where $g$ is the mean dynode secondary emission coefficient (in our case about 3.9 ), and $m=12$ is the number of dynodes in the PMT. The first approximate sign in the above equation is due to the assumption of uniform gain across all dynodes whereas the second one is a result of $g^{m} \gg 1$ for $m=12$.

\subsection{Cross checks}

In order to establish confidence in the analysis method, a set of measurements was carried out to verify the absolute magnitude of $N_{p e}$ and to investigate systematic errors that could affect the interpretation of the results. The same setup was used as that described in Section 3.1, with the only difference being 
that the source and trigger PMT were in turn located at different distances from the PMT along the length of the fiber, resulting in different $N_{p e}$ values. The gain is extracted from the plot of the slope of mean $N_{p e}$ versus mean ADC channel. The corresponding fit is shown in Figure 4 . The behaviour is linear and the extracted slope is $0.183 \pm 0.002$. The y-intercept is consistent with zero $(-0.036 \pm 0.095)$, as expected, demonstrating that there is no systematic (offset) error in the fit and, moreover, that the gain is constant over the the entire range measured in the ADC.

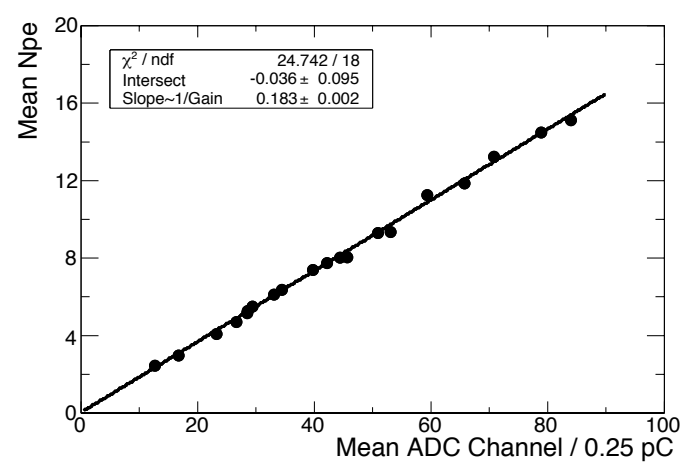

Figure 4: A linear fit to the mean $N_{p e}$ as a function of the mean ADC channel is shown, with the slope being proportional to the inverse gain. The fit passes through the origin, as expected if there is no systematic offset.

The next set of measurements was carried out in order to check the PMT's gain extracted from our $N_{p e}$ measurements against this PMT's gain calibration curve measured by Hamamatsu ${ }^{8}$ as the ratio of the anode to photocathode currents. The PMT gain $\left(g_{P M T}\right)$ in our measurements was derived from the ratio of the anode charge $\left(Q_{\text {anode }}\right)$ - measured in the $\mathrm{ADC}$ - and the number of photoelectrons as follows:

$$
g_{P M T}=Q_{\text {anode }} /\left(e \cdot N_{p e}\right)=(2 \cdot \overline{A D C} \cdot \alpha) /\left(e \cdot N_{p e}\right)=\left(2 \cdot k_{2} \cdot \alpha\right) / e
$$

where $\overline{A D C}$ is an average value of the ADC spectrum, $\alpha=0.25 \mathrm{pC} / \mathrm{ch}$ is the input sensitivity of the LeCroy 2249A ADC used, $e$ is the electron charge, and the factor two in the numerator of the ratio reflects the fact that the ADC (with $50-\Omega$ input impedance) sees only one half of the PMT anode charge because of the employed 50- $\Omega$ termination on the PMT divider. In order to be consistent with the conditions of the R329-02 PMT calibration carried out by Hamamatsu, a Hamamatsu-recommended linear HV divider was used with the ratio of voltage drops between the dynodes being 4:1:1.4:1: .. : 1 . This is in contrast to the fiber

${ }^{8}$ From the calibration sheet provided with the PMT by the manufacturer. 
measurements, where a standard progressive divider was used. The PMT gain results obtained as a function of high voltage are shown in Figure 5 as solid circles and are in close agreement with the calibration curve (solid line) from Hamamatsu.

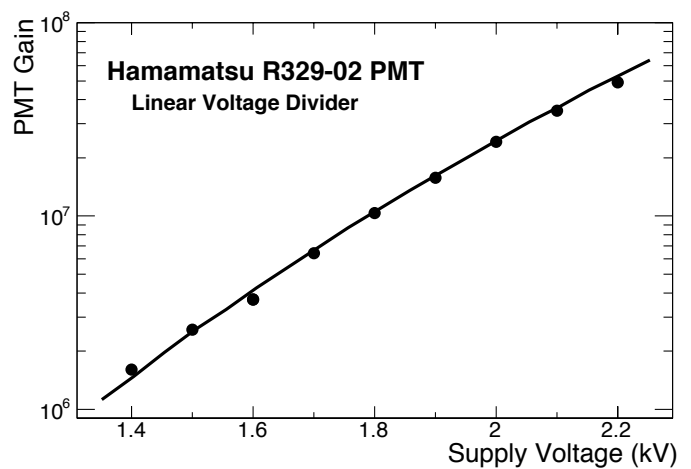

Figure 5: The gain of the R329-02 PMT extracted from our $N_{p e}$ values (solid circles) is plotted as a function of supplied high voltage and compared to Hamamatsu's gain calibration curve (solid line) for this PMT. The error bars are contained within the size of the markers.

Next, a few runs were taken with a silicon photomultiplier (SiPM) opposite to the PMT. The low light conditions from the ${ }^{90} \mathrm{Sr}$ source and fiber afforded clear separation of the photopeaks in the spectrum of the SiPM, thus lending visual confirmation of the extracted average $N_{p e}$ from the SiPM. That number could then be compared to the corresponding number from the PMT as follows:

$$
N_{p e}(P M T)=N_{p e}(S i P M) \cdot \frac{C Q E(P M T)}{P D E(S i P M)} .
$$

The PDE of this SiPM had been previously extracted versus a Hamamatsu S2281 photodiode and was measured to be $(8.2 \pm 0.9) \%$ [ 6 .

To measure the SiPM ADC spectrum, a fiber had both ends polished and was inserted into the dark box. One end was coupled to a SensL ${ }^{9}$ SiPM having a $3 \times 3 \mathrm{~mm}^{2}$ active area, based on their A20HD microcell, and the other end to the PMT. Optical grease was used on both ends. The ${ }^{90} \mathrm{Sr}$ source and trigger counter were positioned at several locations along the length of the fiber, keeping their respective geometry fixed. The collected SiPM ADC spectra displayed individual photoelectron peaks prominently, sometimes visible up to 16 in number. Clear separation of photoelectron peaks in the SiPM ADC spectra allowed a fit function that is the sum of Gaussian peaks; the number of events in each of the $i$-photoelectron peaks, $A_{i}^{s}$, were found from the amplitude and

${ }^{9}$ SensL, Blackrock, Cork, Ireland (www.sensl.com) 


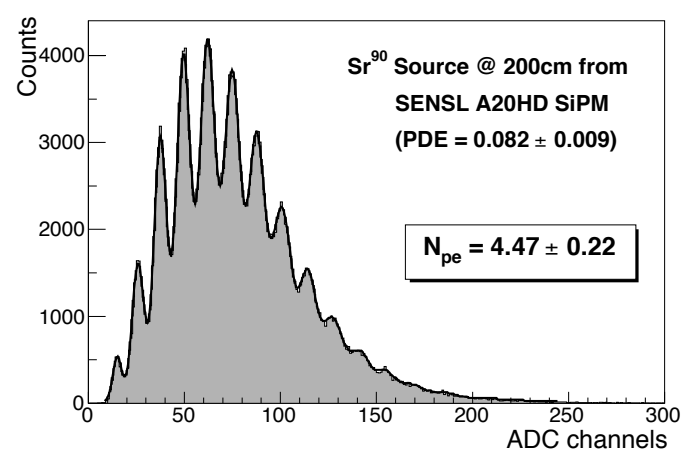

Figure 6: The number of photoelectrons as measured by the SiPM is shown when the ${ }^{90} \mathrm{Sr}$ source and trigger counter is placed at a distance of $200 \mathrm{~cm}$.

width of the corresponding Gaussian peak, and the average $N_{p e}$ was extracted via the formula:

$$
N_{p e}=\frac{1}{A_{s}} \sum_{i}\left(i A_{i}^{s}\right)-\frac{1}{A_{p}} \sum_{i}\left(i A_{i}^{p}\right)
$$

where $A_{s}=\sum A_{i}^{s}$ is the total number of events in the spectrum and the second term on the right-hand side of the equation addresses the contribution of the non-negligible SiPM noise; the total number of "pedestal" events, $A_{i}^{p}$, and the number of events for each of the $i$-photoelectron peaks, $A_{i}^{s}$, was obtained from fitting the pedestal spectrum (viz., the bias voltage is applied to the SiPM, but the ${ }^{90} \mathrm{Sr}$ source is removed). A sample spectrum — following pedestal subtraction - is shown in Figure 6, when the trigger was placed near the middle of the fiber. By using equation 6 and the above numbers for the PDE and CQE with their errors, the average $N_{p e}$ from the SiPM $(4.47 \pm 0.22)$ translates to $(10.8 \pm 1.4)$ for the PMT, a value which is consistent to within one $\sigma$ from the mean extracted from the measurements of all fibers, as reported below in Section 4 .

In the end, the PMT was used for the measurements because its setup was considerably more robust and easy to work with over the period of the project (nearly two years) and the reliability of such PMTs has been established over long periods as opposed to any similar evaluations of SiPMs. Indeed, as the fiber evaluation took place over a period of 22 months, the PMT was checked for signs of degradation: the number of photoelectrons was remeasured $\left(N_{p e}^{\prime}\right)$ from a small set of fibers from shipments 1, 5, 8, 9, 13, 17 (around 30 fibers per shipment). If the PMT sensitivity degraded as a function of time, the ratio $N_{p e}^{\prime} / N_{p e}$ should have become progressively lower than 1 ; this was not observed. 


\section{Results and discussion}

The quality of the resultant $N_{p e}$ fits is demonstrated in Figure 7. where the $\chi^{2} / n d f$ is histogrammed for all fiber measurements. The average value is around 1.4 , and the distribution exhibits a tight grouping with an RMS of $7.8 \%$.

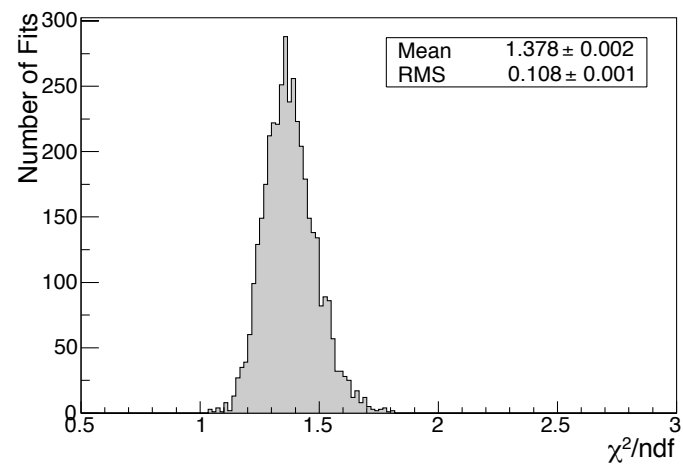

Figure 7: The reduced $\chi^{2} / n d f$ distribution is histogrammed for all fiber fits. The $7.8 \%$ RMS demonstrates the quality of the data acquired as well as the stability of the fits.

The results of the fit extractions for $N_{p e}$ are shown in Figure 8 . The reader is reminded that only fibers from lots $96-707$ (shipments 4-29) were analyzed; lots 1-95 (shipments 1-3) were excluded from this comparison due to different handling of the fiber ends, as mentioned previously. The results from every fiber fit are shown in the top panel of this figure, whereas, in the bottom panel, the results were averaged for each lot with the error bar being the RMS of those distributions. Clearly, there is lot-to-lot variation apparent in the number of photoelectrons as was also manifest in the attenuation length measurements [1. Kuraray observed a similar behaviour in measurements of their own, with the most likely candidate for the difference being small impurities in the secondary dye in the fiber's chemistry, which cannot be controlled in the manufacturing process 12. Light production and transmission are complex processes, strongly dependent on the fiber chemistry of the scintillating material and dopants (dyes), which can affect $N_{p e}$ and attenuation length in different ways. Information on the dye batches was kindly provided to us by Kuraray.

The correlation between the number of photoelectrons and the attenuation length is demonstrated in Figures 9 and 10. It should be noted that the data were selected to bring them to a common footing so as to generate these plots, because the two sets of measurements were conducted independently at separate stations. Due to the large number of fibers tested, the time required for each test, and the need to process the tests quickly in order to keep up with the construction of the BCAL, the measured lots at the two stations differed. For example, a particular lot in one station may have been skipped for reasons of time but may have been measured by the crew on the other station. Only 

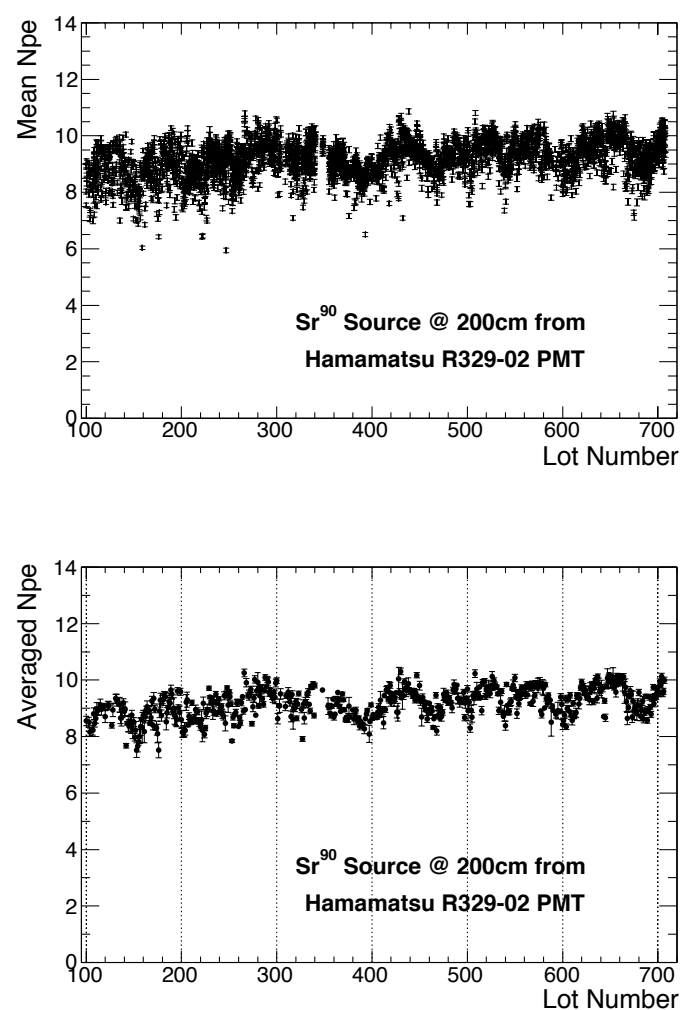

Figure 8: Top panel: The $N_{p e}$ results are plotted for all fibers as a function of lot number (96707), corresponding to shipments 4-29. Multiple fibers were measured from each lot. Bottom panel: The $N_{p e}$ results are averaged by lot number and their average is graphed against the lot number.

the data from lots measured at both stations are depicted in these figures, in contrast to all other figures that contain the full data sets.

Two correlated groupings (clusters) are discernible in the two-dimensional representation and these are plotted separately for dye batch ' $\mathrm{B}$ ' (left panel) and the combined dye batches ' $\mathrm{C}$ ' to ' $\mathrm{F}$ ' (right panel) in Figure 9. The latter are grouped rather tightly and as such are presented as a single group rather than plotted separately. Note that dye batch 'A' corresponds to the fibers that had one end polished (shipments 1-3 or lots 1-95) and where not included in the analysis as explained above.

The dye batch ' $\mathrm{B}$ ' data were then plotted in the top panel of Figure 10, in order to overlay the attenuation length (left vertical axis) and $N_{p e}$ (right vertical axis) curves as a function of lot number (x-axis). Dye batch ' $\mathrm{B}$ ' was used by Kuraray up to lot number 250. The tracking of $N_{p e}$ and attenuation length data is evident with a few exceptions: on average, they both rise or fall in a similar 

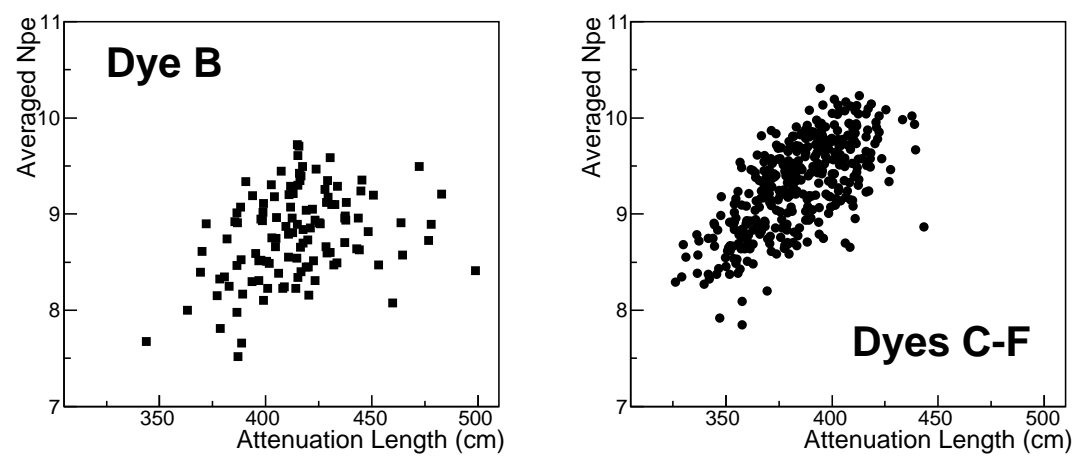

Figure 9: The correlation is shown between $N_{p e}$ and attenuation length for dye batch 'B' (top panel) and for the combined dye batches ' $\mathrm{C}$ ' to ' $\mathrm{F}$ ' (bottom panel). Details are presented in the text.

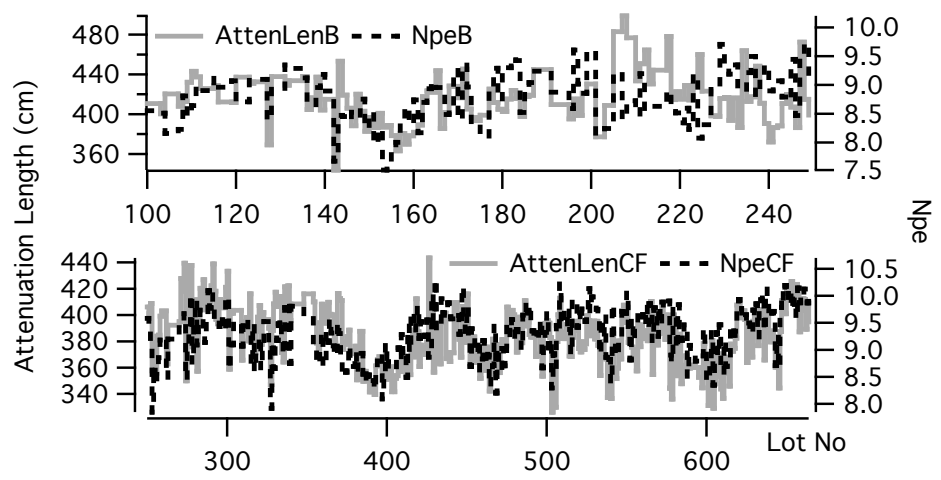

Figure 10: The comparison of distributions of the attenuation length and $N_{p e}$ as a function of fiber lot number is presented for dye batch ' $\mathrm{B}$ ' (top panel) and combined dye batches ' $\mathrm{C}$ ' to 'F' (bottom panel). Details are available in the text.

manner. This is expected as the $N_{p e}$ was extracted after the light traveled $200 \mathrm{~cm}$ along the fiber and thus its value is affected by the attenuation length, which in turns depends on the fiber chemistry. The same tracking is apparent (and to greater clarity due to the larger number of lots) in the batch 'C-F' data, presented on the bottom panel. Separate plots were generated primarily to allow for different scaling coefficients in the overlay of the $N_{p e}$ and attenuation length curves for the two dye groupings. Clearly something changed in the chemistry between dye ' $\mathrm{B}$ ' and the remaining dyes, and this explains the need for two separate scale factors and also that the two groupings are separately located in different regions of the two-dimensional plots in Figure 9.

The final results of all $N_{p e}$ data are histogrammed in the top panel of Figure 11. The mean and standard deviation were extracted to be $9.17 \pm 0.06$ 
$(6.6 \%)$. The contract specifications with Kuraray included a term that the overall RMS had to be less than $15 \%$, which was satisfied by $98 \%$ of the fibers measured based on our $6.6 \% \sigma$. Furthermore, the spread within each lot is histogrammed in the bottom panel of the same figure and is on average $4 \%$, which is significantly less that the overall spread of $6.6 \%$ : the difference (about $5.3 \%$ ) between these extracted values is most likely due to variations in the fiber chemistry during the manufacturing process across preforms (lots). This encompasses the uncertainties in the experimental (fiber polishing and coupling, etc.) and analysis methodology as well as variations in the fiber chemistry during the manufacturing process. Finally, no fibers resulted in $N_{p e}<4.5$ and fewer than 10 gave $4.5<N_{p e}<6.0$, a testament to their quality.
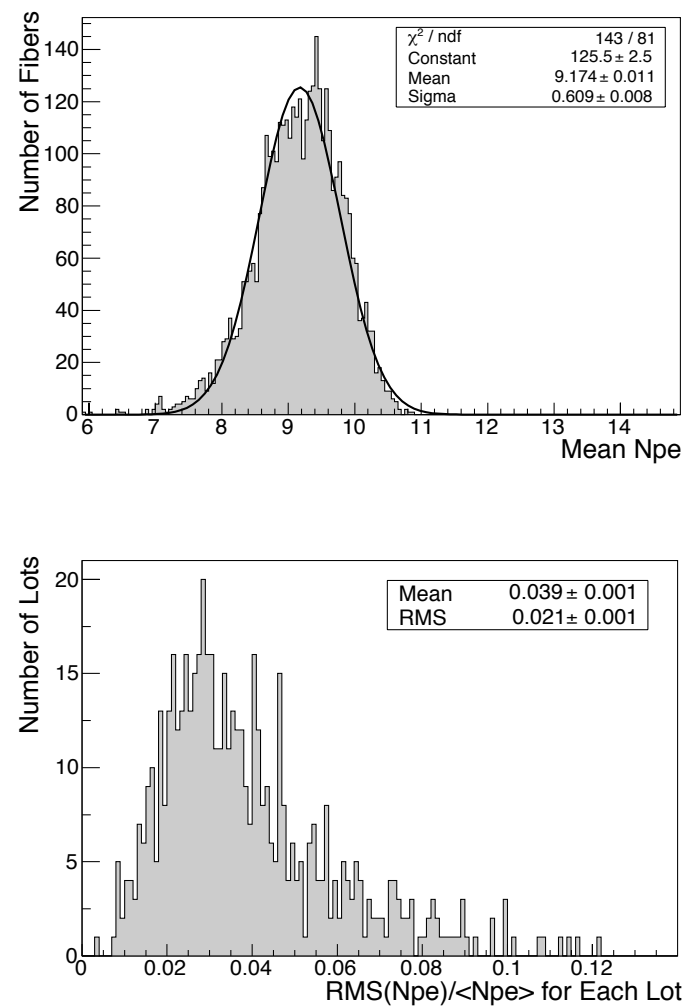

Figure 11: Top panel: The $N_{p e}$ results from all measured fibers are histogrammed and fitted using a Gaussian function. Bottom panel: The spread in the fits for each lot is expressed as the ratio of the RMS divided by the average $N_{p e}$ for the lot: $R M S\left(N_{p e}\right) /\left\langle N_{p e}\right\rangle$. This ratio is histogrammed and demonstrates that the fiber measurements within a lot are typically spread to a few percent. 


\section{Summary and conclusions}

The objective of this study was to characterize the Kuraray SCSF-78MJ scintillating fibers in terms of their light yield. In the process, we have developed a robust analysis method in extracting $N_{p e}$ in cases where individual photoelectron peaks are not discernible in the acquired spectra, such as when using ordinary bialkali PMTs or large-area silicon photomultipliers. The method is based on simulating the energy deposition responsible for the production of photons in a fiber using a Poisson function to describe the distribution of the photons on the photocathode of a calibrated PMT, handling the conversion of photons to photoelectrons through a Binomial distribution - which in the end was approximated by a Poisson to keep the analysis time manageable and, finally, capturing the process of electron multiplication through the PMT's dynode chain by a Gaussian distribution. In the process, the extraction of the PMT's gain was verified against the manufacturer's measurements and cross checks revealed no systematic offsets in the extraction of $N_{p e}$ beyond the quoted error of the measurement.

The resulting data set shows that there is lot-to-lot variation apparent in the extracted $N_{p e}$ similar to what our group had observed in previous measurements of the attenuation length of these fibers and this effect is correlated in the two distributions. The observed features are mostly a function of the fiber chemistry (preform batch dye) but are also dependent on the additional errors from both experimental and analysis sides. This explains why measurements on a single fiber, or even an entire batch, may result in numbers differing within a range.

The contract specifications by the GlueX Collaboration were as follows: the number of photoelectrons $\left(N_{p e}\right)$ collected at the fiber's end had to be greater than 4.5 using a bialkali PMT at $200 \mathrm{~cm}$ from the source with $R M S \leq 15 \%$. The mean extracted number of photoelectrons at $200 \mathrm{~cm}$ was found to be 9.17 , comfortably above the requirement. The RMS of the $N_{p e}$ distribution was $6.6 \%$, thus containing $98 \%$ of the fibers within the contract ceiling of $15 \%$. Finally, the average number of $9.17 \pm 0.6$ was found to be consistent with a measurement using a SiPM where the individual photopeaks were visible that, if anything, yielded an even higher $N_{p e}$ number of $10.8 \pm 1.4$.

\section{Acknowledgments}

This work was supported by NSERC grant SAPJ-326516 and DOE grant DE-FG02-0SER41374, and by Jefferson Science Associates, LLC, who operate Jefferson Lab under U.S. DOE Contract No. DE-AC05-06OR23177. We wish to thank Kuraray for providing us with their detailed data. We also acknowledge the input of Y. Qiang, C. Zorn, E. Chudakov, F. Barbosa and B. Zihlman at Jefferson Lab, and the assistance of D. Kolybaba and K. Wolbaum at the University of Regina Faculty of Science Machine and Electronics Shops. 


\section{Figure captions}

Fig. 1. The geometrical setup used to measure the number of photoelectrons from the fibers is sketched (not to scale); the PMTs, fiber and source are enclosed in a $4.5 \mathrm{~m}$ long dark box. The inset on the left shows the acrylic piece that is used to guide the fiber in contacting the PMT, and the inset on the right shows the cross sectional view of the lead collimator and its lid containing the ${ }^{90} \mathrm{Sr}$ radioactive source. The circuit diagram is also presented. The reader is referred to the text for more details.

Fig. 2. The simulated distribution of the energy deposition in a fiber is shown. The simulated mean energy deposition was $\sim 0.19 \mathrm{MeV} /$ fiber, corresponding to traversal of $\beta$ particles from the ${ }^{90} \mathrm{Sr} /{ }^{90} \mathrm{Y}$ mother/daughter pair past the collimator and through the 1-mm fiber. The simulated geometry mirrors the experimental setup as described in the text.

Fig. 3. A typical photoelectron spectrum is shown when both the ${ }^{90} \mathrm{Sr}$ source and the trigger counter were positioned $200 \mathrm{~cm}$ from the fiber's end. The spectrum is fit with a Poisson function with the extracted mean number of photoelectrons and the $\chi^{2}$ per d.o.f. displayed in the inlay. Details on the fitting function are described in the text.

Fig. 4. A linear fit to the mean $N_{p e}$ as a function of the mean ADC channel is shown, with the slope being proportional to the inverse gain. The fit passes through the origin, as expected if there is no systematic offset.

Fig. 5. The gain of the R329-02 PMT extracted from our $N_{p e}$ values (solid circles) is plotted as a function of supplied high voltage and compared to Hamamatsu's gain calibration curve (solid line) for this PMT. The error bars are contained within the size of the markers.

Fig. 6. The number of photoelectrons as measured by the SiPM is shown when the ${ }^{90} \mathrm{Sr}$ source and trigger counter is placed at a distance of $200 \mathrm{~cm}$.

Fig. 7. The reduced $\chi^{2} / n d f$ distribution is histogrammed for all fiber fits. The $7.8 \%$ RMS demonstrates the quality of the data acquired as well as the stability of the fits.

Fig. 8. Top panel: The $N_{p e}$ results are plotted for all fibers as a function of lot number (96-707), corresponding to shipments 4-29. Multiple fibers were measured from each lot. Bottom panel: The $N_{p e}$ results are averaged by lot number and their average is graphed against the lot number.

Fig. 9. The correlation is shown between $N_{p e}$ and attenuation length for dye batch 'B' (top panel) and for the combined dye batches ' $\mathrm{C}$ ' to ' $\mathrm{F}$ ' (bottom panel). Details are presented in the text.

Fig. 10. The comparison of distributions of the attenuation length and $N_{p e}$ as a function of fiber lot number is presented for dye batch 'B' (top panel) and combined dye batches ' $\mathrm{C}$ ' to ' $\mathrm{F}$ ' (bottom panel). Details are available in the text.

Fig. 11. Top panel: The $N_{p e}$ results from all measured fibers are histogrammed and fitted using a Gaussian function. Bottom panel: The spread in the fits for each lot is expressed as the ratio of the RMS divided by the average 
$N_{p e}$ for the lot: $R M S\left(N_{p e}\right) /\left\langle N_{p e}\right\rangle$. This ratio is histogrammed and demonstrates that the fiber measurements within a lot are typically spread to a few percent.

\section{References}

[1] A.E. Baulin et al., Nucl. Instr. and Meth. A 715 (2013) 48-55.

[2] V. Crede et al. (The GlueX Collaboration), "An initial study of mesons and baryons containing strange quarks with GlueX", Proposal to the Jefferson Lab Program Advisory Committee \#40, GlueX Note GlueX-doc-2198 (2013).

[3] F. Barbosa, J.E. McKisson, J. McKisson, Y. Qiang, E. Smith, C. Zorn, Nucl. Instr. and Meth. A 695 (2012) 100-104.

[4] Y. Qiang, C. Zorn, F. Barbosa and E. Smith, Nucl. Instr. and Meth. A 698 (2013) 234-241.

[5] O. Soto, R. Rojas, S. Kuleshov, H. Hakobyan, A. Toro, W.K. Brooks, R. Rios, Nucl. Instr. and Meth. A 739 (2014) 89-97.

[6] K. Janzen, A. Semenov, G.J. Lolos and Z. Papandreou, GlueX Collaboration Technical Report, GlueX-doc-1178 (2008).

[7] A. Fasso, A. Ferrari, J. Ranft, P.R. Sala, "FLUKA: a multi-particle transport code", CERN-2005-10 (2005), INFN/TC05/11, SLAC-R-773.

[8] I. Chirikov-Zorin et al., Nucl. Instr. Meth. A 456 (2001) 310-324.

[9] R. Bollmann et al. Nucl. Instr. Meth. A 342 (1994) 466-472.

[10] A. Semenov, Z. Papandreou, I. Semenova, GlueX Collaboration Technical Report, GlueX-doc-845 (2007).

[11] Z. Papandreou, B.D. Leverington, G.J. Lolos, Nucl. Instr. and Meth. A 596 (2008) 338-346.

[12] Kuraray, private communication (2011). 